\title{
Circulating Levels of Thrombospondin-1 and Thrombospondin-2 in Patients with Temporal Lobe Epilepsy Before and After Surgery
}

\author{
Orkhan ALIZADA ${ }^{1}$, Mehmet Yigit AKGUN¹, Ahmet Faruk OZDEMIR ${ }^{1}$, Sureyya TOKLU ${ }^{1}$, Rahsan KEMERDERE ${ }^{1}$, \\ Bagnu ORHAN ${ }^{2}$, Berrin Bercik INAL ${ }^{1}$, Seher Naz YENI ${ }^{3}$, Taner TANRIVERDI ${ }^{1}$ \\ ${ }^{1}$ Istanbul University-Cerrahpasa, Cerrahpasa Medical Faculty, Department of Neurosurgery, Istanbul, Turkey \\ ${ }^{2}$ Istanbul Training and Research Hospital, Clinical Biochemistry Laboratory, Istanbul, Turkey \\ ${ }^{3}$ Istanbul University-Cerrahpasa, Cerrahpasa Medical Faculty, Department of Neurology, Istanbul, Turkey
}

Corresponding author: Taner TANRIVERDI tanerato2000@yahoo.com

\section{ABSTRACT}

AIM: To measure the serum levels of strong angiostatic and synaptogenetic molecules thrombospondin-1 (TSP-1) and thrombospondin-2 (TSP-2) in patients with temporal lobe epilepsy (TLE) before and after surgery.

MATERIAL and METHODS: In this prospective study, 20 patients operated for TLE and 20 healthy subjects were included. Serum levels of TSP-1 and TSP-2 were measured using enzyme-linked immunosorbent assay (ELISA).

RESULTS: Our findings showed that both groups had higher serum levels of both molecules "before" surgery than 10 days "after" surgery. However, a significant difference was noted between "before" and "after" surgery regarding TSP-1 ( $p=0.00001)$. Although a marked decrease was found "after" surgery with respect to TSP-2, the difference did not reach statistical significance ( $p=0.22)$. In patients with TLE, serum levels of both molecules "before" surgery were found to be significantly higher than in healthy controls (TSP-1, $\mathrm{p}=0.00001$; TSP-2, $\mathrm{p}=0.007$ ).

CONCLUSION: Serum levels of TSP-1 and TSP-2 are determined to be higher in patients with TLE than in healthy subjects, and the resection of epileptogenic tissues decreases the serum levels of these molecules. Future studies should involve a higher number of patients with serial serum levels of TSP-1 and TSP-2 at the long-term follow-up to correlate with seizure outcome.

KEYWORDS: Angiogenesis, Seizure, Synaptogenesis, Temporal Lobe Epilepsy, Thrombospondin

\section{INTRODUCTION}

$\mathrm{T}$ Temporal lobe epilepsy (TLE) has been identified as the most common form of focal epilepsy, and although medical treatment is effective, the majority of patients with TLE must undergo surgical resection. In TLE, one important concern is the recurrence of seizure after extended resection of the temporal lobe. The pathophysiology behind the seizure recurrence despite extensive surgery is confusing because we lack understanding on the pathogenesis of epilepsy; thus, a better understanding on the pathogenesis of epilepsy is of utmost importance to provide new targets for antiepileptic drugs (AEDs) and effective treatment.

Epilepsy is clearly a neural network disease, and seizure is the final expression of the precise balance between functions of inhibitory and excitatory neurons. The propagation of abnormal electrical discharges from the main epileptic focus to distant areas in the brain can cause pathological restructuring of the neural network (4), and increase angiogenesis, which has been shown to be involved in the pathogenesis of epilepsy (7). Clinical and experimental studies have suggested that
Orkhan ALIZADA $\quad$ (1) : 0000-0003-0942-9906

Mehmet Yigit AKGUN (D): 0000-0003-1342-7663

Ahmet Faruk OZDEMIR (D) : 0000-0002-7919-1609
Sureyya TOKLU (10 : 0000-0001-9396-8202

Rahsan KEMERDERE (D): 0000-0002-9947-3486

Bagnu ORHAN
Berrin Bercik INAL (1) : 0000-0002-9098-4140

Seher Naz YENI (1) : 0000-0001-7372-5430

Taner TANRIVERDI : : 0000-0002-1878-0696 
interfering pathological synaptogenesis and angiogenesis inhibits or at least delays the pathological formation of an epileptic network $(1,3,14)$.

Recently, studies have identified that astrocytes are important cells playing essential roles in stabilizing synapsis and in forming pathological angiogenesis by secreting thrombospondins (TSP) $(2,14)$. Thrombospondins have several important functions, and these include propagation of apoptosis, activation of transforming growth factor-beta (TGF- $\beta$ ), and immune regulation (14). More importantly, thrombospondin-1 (TSP-1) and thrombospondin-2 (TSP-2) are regulatory proteins that increase the formation of new synapsis and blood vessels (8). In a mouse model of epilepsy, a significantly reduced number of new synapses were found in TSP-1 knockout mice (6). Thus, increased TSP-1 leads to increased synaptogenesis after seizure in animal models of epilepsy (14), and inhibition of the pathways in which TSP-1 significantly decreased synapse formation and stopped or delayed seizure activities. Accordingly, studies have suggested that TSPs could be strong therapeutic targets especially in epilepsy refractory to other treatments $(6,14)$.

To the best of our knowledge, this study is the first to show circulating serum levels of TSP-1 and TSP-2 in patients with TLE. Given that TSP-1 and TSP-2 play significant roles in synaptogenesis and angiogenesis, which are related to epilepsy, we hypothesized that serum levels of TSP-1 and TSP-2 should be higher in patients with TLE than in normal individuals and that the levels should decrease after surgery.

\section{MATERIAL and METHODS}

In this study, 20 patients who underwent anterior temporal lobectomy (ATL) because of refractory TLE and 20 control subjects were enrolled. Clinical features, surgical/pathological findings, and results of follow-up have been obtained from the patients' records. This propective clinical study was approved by the local ethics committee of Istanbul UniversityCerrahpasa (Approval date: 03.10.2018; No: 68840). All patients and controls were informed about the aim of the study and provided written informed consent before participating in the study. All procedures in this study complied with the Declaration of Helsinki of the World Medical Association.

\section{Study Population}

Routine extensive epilepsy protocol was performed in all patients before surgery. Careful documentation of findings of seizure semiology and neurological examination was performed. Scalp electroencephalography (EEG) and magnetic resonance imaging were routinely obtained. If discordant data were found among noninvasive studies, depth electrode insertion and positron emission tomography scan were performed. Findings from both noninvasive and invasive preoperative diagnostic work-ups were extensively and carefully examined in our local epilepsy meetings held weekly. In case of a surgical indication was made, the risk-benefit ratio was extensively discussed with either the patients or next of kin (5). Surgical intervention was made possible after the patients or next of kin signed the informed consent. ATL was the chosen surgical approach performed in all patients. All patients after surgery were followed up with regular intervals by both surgical and neurological teams with respect to seizure outcome (5).

\section{Sample Collection}

Venous blood samples were obtained from the controls and patients preoperatively and 10 days after surgery. Blood was collected using a Vacutainer ${ }^{\circledR}$ tube (Becton, Dickinson and Company, Franklin Lakes, New Jersey, USA) and centrifuged at $1500 \mathrm{xg}$ for 30 minutes. The serum was then stored at $-80^{\circ}$ C until laboratory analysis.

\section{Biochemical Determination}

In this study, human TSP-1 and TSP-2, which are identified as important markers of angiogenesis and synaptogenesis, were included. The serum levels of TSP-1 and TSP-2 $(\mathrm{ng} / \mathrm{mL})$ were assessed using quantitative sandwich enzyme-linked immunosorbent assay (ELISA) kits (Elabscience Biotechnology Inc., Houston, Texas, USA) according to the manufacturer's instructions.

\section{Statistical Analysis}

Results are expressed in mean \pm standard deviations for continuous variables. Group comparisons were made using the nonparametric "Mann-Whitney U test," and bivariate correlation analysis was performed using "Pearson's correlation" test. All statistical analyses were performed using Statistical Package for Social Sciences, version 20.0 (IBM, Chicago, USA). $p<0.05$ was considered statistically significant.

\section{RESULTS}

\section{Demographic and Clinical Characteristics of Study Subjects}

Twenty patients, consisting of 10 women (50\%) and 10 men (50\%), operated for TLE between January 2016 and June 2019 were included in this study. The mean age was 33.3 \pm 8.0 years. In addition, 20 healthy controls, consisting of 9 women (45\%) and 11 men (55\%) were also enrolled in the study. Their mean age was $34.3 \pm 5.8$ years. No significant differences were found between the patients and controls with respect to age (Mann-Whitney $U$ test; $p=0.43)$ and gender $\left(X^{2}\right.$ test; $p=0.65)$. The onset of seizure was $11.1 \pm 6.9$ years, and duration was $23.3 \pm 9.7$ years. The mean seizure frequency per month was $8.4 \pm 13.7$, and all were treated with an AED. Right- and left-sided resections were performed in 10 (50\%) and $10(50 \%)$ patients, respectively. Five patients $(25 \%)$ have underwent invasive EEG monitoring with depth electrodes to discover the side and site of the epileptogenic temporal lobe. Regarding histopathological diagnosis, type-I and typeII hippocampal sclerosis were diagnosed in 16 (80\%) and 4 (20\%) patients, respectively. Focal cortical dysplasia was noted in the resected temporal cortices in 16 (80\%) patients.

\section{Serum Levels of TSP-1 and TSP-2}

Serum levels of TSP-1 and TSP-2 are summarized in Table I. In patients with TLE, both molecules showed higher serum levels "before" surgery compared to 10 days "after" surgery (Figures 
1, 2). However, a significant difference was found between "before" and "after" surgery regarding TSP-1 (Mann-Whitney $\mathrm{U}$ test; $\mathrm{p}=0.00001)$. Although a marked decrease was found "after" surgery with respect to TSP-2, the difference was not statistically significant (Mann-Whitney $U$ test; $p=0.22$ ). Comparing the serum levels of both molecules between patients with TLE and health controls, "before" surgery, serum levels of TSP-1 (Mann-Whitney $U$ test; $p=0.00001$ ) and TSP-2 (Mann-Whitney $U$ test; $p=0.007$ ) were significantly higher in patients with TLE than in healthy controls. Surgery significantly decreased the serum levels of both molecules in patients with TLE, such that no significant differences were identified between the serum levels of both molecules "after" surgery in patients with TLE and those in controls (TSP-1: Mann-Whitney $\mathrm{U}$ test, $\mathrm{p}=0.35$; TSP-2: Mann-Whitney $\mathrm{U}$ test, $\mathrm{p}=0.08)$.

\section{Correlation Analysis}

The duration of epilepsy and seizure type did not inhibit any correlation with both molecules (nonparametric Spearman's rho; $p>0.0$ ). Seizure frequency showed a tendency toward a negative correlation with TSP-2, but it was not significant (nonparametric Spearman's rho; correlation coefficient: -0.12 ; $p=0.6)$. Meanwhile, a positive correlation was noted between TSP-1 and TSP-2 (nonparametric Spearman's rho; correlation coefficient: 0.59; $p=0.006$ ).

\section{DISCUSSION}

The roles of astrocytes in the development of epilepsy have been understood recently, and their importance has been demonstrated in experimental animal models of epilepsy $(6,9,11,13,14)$. Astrocytes are considered to be the main cells secreting thrombospondins, especially TSP-1 and TSP-2. TSP-1 seems to promote neuronal migration, axonal growth, and more importantly synaptogenesis $(8,10)$. Stimulating excitatory synapses, which play an important role in epilepsy development, is possible with the interaction of TSP-1 and the a2 $\delta-1$ subunit of the gabapentin receptors (12). Epilepsy is a network-based disease, and several lines of evidence indicated that abnormal synaptogenesis contributes to epilepsy and the propagation of abnormal electrical discharges to both the ipsilateral and contralateral brain areas. Studies involving several rat models of epilepsy, such as amygdala-kindling (11) or kainic acid-induced status epilepticus models (13), have demonstrated that the increasing number of new excitatory synapses caused by TSP-1 contributes to the development of a hyperexcitable neuronal network, which is a sine qua non in epilepsy.

Consistent with the previous results from animal models of epilepsy $(6,9,11,13,14)$, we, for the first time, found high serum levels of TSP-1 and TSP-2 in patients with TLE compared with normal subjects. Depending on the experimental

Table I: Mean ( \pm Standard Deviation) Serum Levels $(\mathrm{ng} / \mathrm{mL})$ of Human Thrombospondin-1 (TSP-1) and Thrombospondin-2 (TSP-2) in Patients (Before and After Surgery) and Controls

\begin{tabular}{cccr}
\hline Parameters & Before surgery & After surgery & Controls \\
\hline TSP-1 & $57282.4 \pm 14903.4$ & $26970.6 \pm 19138.2$ & $19045.08 \pm 6314.08$ \\
\hline TSP-2 & $12.06 \pm 15.9$ & $5.52 \pm 4.8$ & $3.40 \pm 1.15$ \\
\hline
\end{tabular}

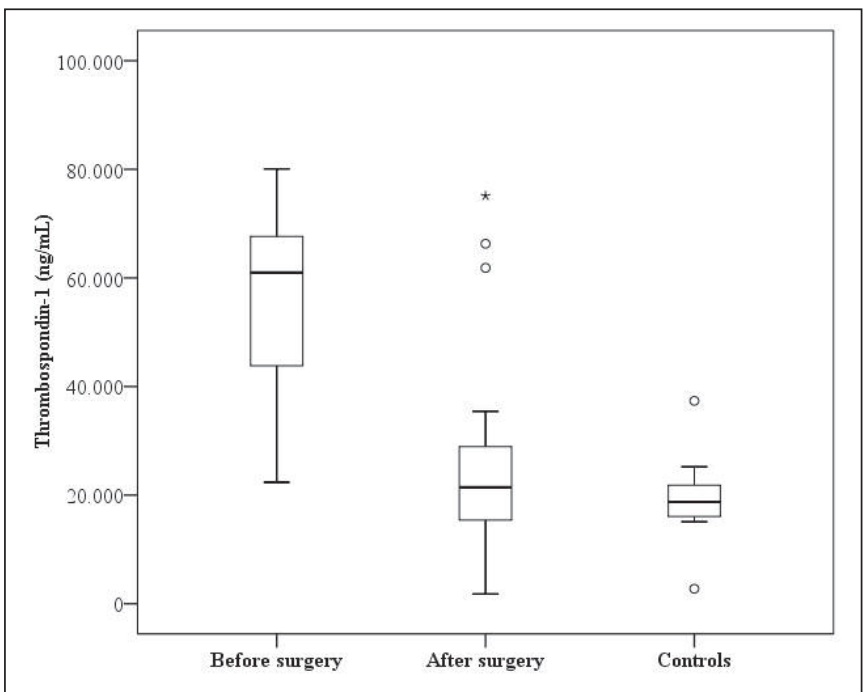

Figure 1: Graph showing serum levels of thrombospondin-1 in patients and controls. Patients showed significant higher serum levels before surgery compared to the controls $(p=0.00001)$. Also surgery caused significant decrease in serum levels $(p=0.00001)$.

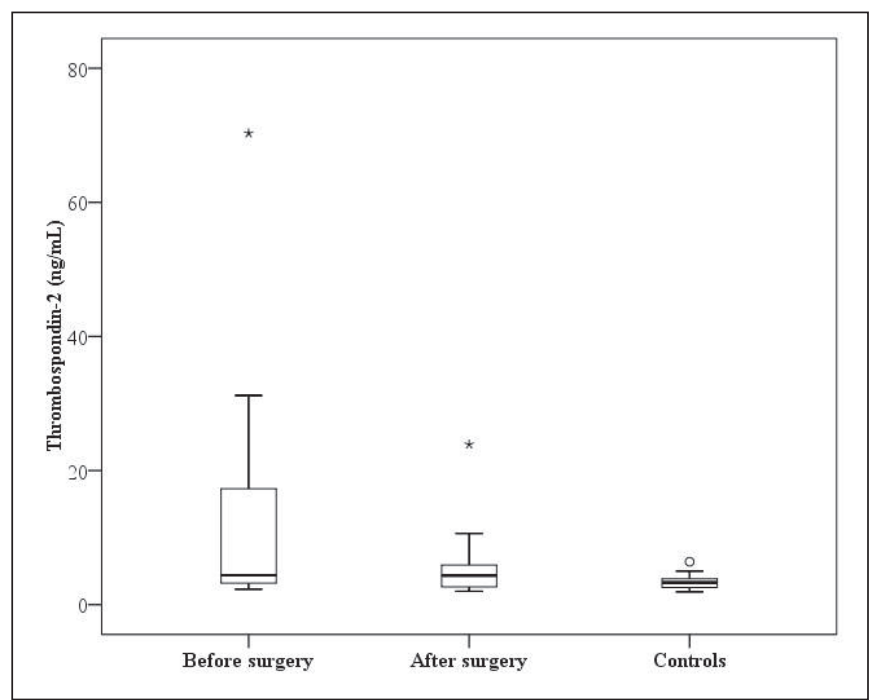

Figure 2: Graph showing serum levels of thrombospondin-2 in patients and controls. Patients showed significant higher serum levels before surgery compared to the controls $(p=0.007)$. Also surgery caused decrease in serum levels but the difference did not reach significant levels $(p=0.22)$. 
results, we simply hypothesized that TSP-1 (more important in the development of new excitatory synapses than TSP-2) and TSP-2 should be higher in patients with TLE compared to normal subjects and removal of epileptogenic tissues (temporal lobe resection) should decrease the serum levels of both molecules. Our results proved these hypotheses. The decrease in TSP-1 levels was significant that no difference was found between the controls and patients after surgery. The decrease in the serum levels of TSP-2 after surgery was also found in patients with TLE with, however, an insignificant difference from the controls. We should emphasize that if we could include a higher number of patients, the difference between the controls and patients with TLE after surgery would be significant.

Our results conform to the results of a limited number of animal studies $(6,9,11,13,14)$. An important point that readers should pay attention to is that TSP levels in animal models of epilepsy were obtained after acute seizures; however, we evaluated the serum levels of TSP in humans with chronic epilepsy. Thus, our results should be evaluated carefully. Sun et al., in their study involving amygdala-kindling rat models of epilepsy, have provided the first evidence of increased levels of TSP-1 with increased density of synapses and excitatory synaptic markers in the hippocampus using immunohistochemistry tests (11). More importantly, they further showed that inhibiting TSP-1 functions reduced the synaptic number, delayed the progression of epilepsy, and diminished the severity of and the susceptibility to kindling. In their study using kainic acid-induced status epilepticus animal models, Zhang et al. demonstrated that increased TSP- 1 expression, TGF- $\beta 1$ expression, and pSmad2/3 levels in both the hippocampus and temporal cortex were found (14). Inhibiting TSP-1 expression with siRNA and inhibiting TGF- $\beta 1$ activation were found to significantly attenuate seizure severity. They also found that in the siRNA group, inhibiting TSP-1 decreased vessel density and increased the permeability of the bloodbrain barrier in comparison with the control anımals. Their results have indicated that inhibiting TSP-1 activity decreased angiogenesis and the severity of acute seizures. In contrast to our results and other animal studies, a reduction in TSP1 expression has been shown in the ventrobasal nucleus of the thalamus in a rat model of spontaneous absence epilepsy and in patients with generalized epilepsy (9). Furthermore, increased sensitivity of pentylenetetrazol-induced kindling has been reported in mice lacking TSP-1 (6). This result indicated that TSP-1 plays different roles in different phases of epilepsy, and reports have suggested that both reduced and elevated TSP-1 levels can aggravate seizures.

Depending on our results and the results of a limited number of animal studies on epilepsy, we speculate that TSP-1 and TSP-2 both play an important role in the development of TLE and propagation of seizures. The reduction in the serum levels of TSP-1 and TSP-2 in our study suggested that resection of epileptic tissues has decreased the excitatory synapses, which is considered a critical event in epilepsy.

\section{CONCLUSION}

Serum levels of TSP-1 and TSP-2 are determined to be higher in patients with TLE than in healthy subjects, and the resection of epileptogenic tissues is seen to decrease the serum levels of both molecules. Given that the important roles of both molecules, especially TSP-1, in the development and progression of epilepsy have been proven, our results in humans support the hypotheses that TSPs play an important role in the development of TLE and inhibition of the pathways at one point in which TSP-1 or TSP-2 is a key molecule could significantly inhibit epileptic activity and its spread and provide novel therapeutic targets for treating epilepsy. Future studies should include a higher number of patients with serial serum levels of TSP-1 and TSP-2 at the long-term follow-up to correlate with seizure outcome.

\section{ACKNOWLEDGEMENTS}

Orkhan Alizada, Mehmet Yigit Akgun, Ahmet Faruk Özdemir, Sureyya Toklu: Assisted the surgeries and got the biological samples and collected the data related to the patients and controls. Taner Tanriverdi and Rahsan Kemerdere: Operated on the patients and planned the study and wrote the article. Bagnu Orhan and Berrin Bercik Inal: Keep the materials in biochemistry laboratory and performed biochemistry studies. Seher Naz Yeni: Followed-up the patients as the chief epileptologist.

\section{REFERENCES}

1. Benini R, Roth R, Khoja Z, Avoli M, Wintermark P: Does angiogenesis play a role in the establsihment of mesial temporal lobe epilepsy? Int J Dev Neurosci 49:31-36, 2016

2. Crawford DC, Jiang X, Taylor A, Mennerick S: Astrocytederived thrombospondins meadiate the development of hippocampal presynaptic plasticity in vitro. J Neurosci 32:13100-13110, 2012

3. Croll SD, Ransohoff RM, Cai N, Zhang Q, Martin FJ, Wei T, Kasselman LJ, Kintner J, Murphy AJ, Yancopoulos GD, Wiegand SJ: VEGF-mediated inflammation precedes angiogenesis in adult brain. Exp Neurol 187:388-402, 2004

4. Heller JP, Rusakov DA: Morphological platicity of astroglia: Understanding synaptic microenvironment. Glia 63:21332151, 2015

5. Kemerdere R, Ahmedov ML, Alizada O, Yeni SN, Oz B, Tanriverdi T: Effect of temporal neocortical pathology on seizure freeness in adult patients with temporal lobe epilepsy. World Neurosurg 116:e801-e805, 2018

6. Mendus D, Rankin-Gee EK, Mustapha M, Porter BE: Increased sensitivity to kindling in mice lacking TSP1. Neurosci 305:302308, 2015

7. Morin-Brureau M, Rigau V: Why and how to get angiogenesis in focal epilepsies. Epilepsia 6:64-68, 2012

8. Risher WC, Eroglu C: Thrombospondins as key regulators of synaptogenesis in the central nervous ssytem. Matrix Biol 31:170-177, 2012 
9. Santolini I, Celli R, Cannella M, Imbriglio T, Guiducci M, Parisi $\mathrm{P}$, Schubert J, lacomino M, Zara F, Lerche H: Alterations in the a2 $\delta$ ligand, thrombospondin-1, in a rat model of spontaneous absence epilepsy and in patients with idiopathic/genetic generalized epilepsies. Epilepsia 58:1993-2001, 2017

10. Stenina-Adognravi O: Invoking the power of thrombospondins: Regulation of thrombospondins expression. Matrix Biol 1:6982, 2014

11. Sun H, Ma L, Zhang Y, Pan X, Wang C, Zhang J, Zhang X, Sun $H$, Wang $Q$, Zhu W: A purinergic $P 2$ receptor family-mediated increase in thrombospondin-1 bolsters synaptic density and epileptic seizure activity in the amygdala-kindling rat model. Front Cell Neurosci 12:302-313, 2018
12. Vartanian MG, Radulovic LL, Kinsora JJ, Serpa KA, Vergnes M, Bertram E, Taylor CP: Activity profile of pregabalin in rodent models of epilepsy and ataxia. Epilepsy Res 68:189205, 2006

13. Zhang $Y$, Zhang $M$, Zhu W, Pan X, Wang Q, Gao X, Wang C, Zhang X, Liu Y, Li S, Sun H: Role of elevated thrombospondin-1 in kainic acid-induced status epilepticus. Neurosci Bull 36(3):263-276, 2019

14. Zhang Y, Zhu W, Yu H, Yu J, Zhang M, Pan X, Gao X, Wang Q, Sun H: P2Y4/TSP-1/TGF- $31 / p S m a d 2 / 3$ pathway contributes to acute generalized seizures induced by kainic acid. Brain Res Bull 149:106-119, 2019 